\title{
Perineurioma: an uncommon lesion in the gastrointestinal tract
}

A 45-year-old man was referred to our hospital with a gastric nodule. Gastroscopy revealed a flat, pale-colored lesion, approximately $15 \mathrm{~mm}$ in diameter, in the gastric body. The lesion showed some nodularity but seemed to be covered with a slightly inflamed mucosa ( $\bullet$ Fig. 1). Endoscopic ultrasound (EUS) showed a thickened mucosal layer, as well as scattered, small hypoechoic areas in the submucosal layer ( $\bullet$ Fig. 2).

A biopsy was taken but the final diagnosis was not definitive because of the small size of the samples. Endoscopic submucosal dissection (ESD) was performed to obtain a precise diagnosis, as well as for removal of the lesion. Histopathologically, the lesion consisted of a focal proliferation of spindle cells without atypia that were present from the lamina propria to the submucosa ( Fig.3a). Immunohistochemistry revealed that the spindle cells were positive for EMA and claudin-1 ( $\bullet$ Fig.3b,c). Therefore, this lesion was eventually diagnosed as a gastric perineurioma with no evidence of malignancy.

Benign peripheral nerve sheath tumors, which occur uncommonly in the gastrointestinal tract, include ganglioneuromas, neurofibromas, and schwannomas. Perineuriomas are rare, benign peripheral nerve sheath tumors that include soft tissue, intraneural, and sclerosing variants [1]. The first soft tissue perineurioma was described by Lazarus and Trombetta in 1978 as an intramuscular neurofibromalike tumor of the calf with ultrastructural features characteristic of perineurial cells [2]. To date, there are only two case reports of gastric perineuriomas $[3,4]$.
It is important to emphasize that distinguishing perineuriomas from other spindle cell neoplasms of the gastrointestinal tract can be facilitated by immunostaining for EMA and claudin-1. Agaimy and Wuensch suggested that this exceedingly rare mesenchymal neoplasm might have been under-recognized or misinterpreted as a gastrointestinal stromal tumor (GIST) before the era of KIT immunostaining. Perineurioma should therefore be included in the differential diagnosis of spindle cell GISTs with unusual features, especially the so-called KIT-negative GISTs. Gastroenterologists, as well as pathologists, should be aware of this type of submucosal lesion.

Endoscopy_UCTN_Code_CCL_1AB_2AD_3AB

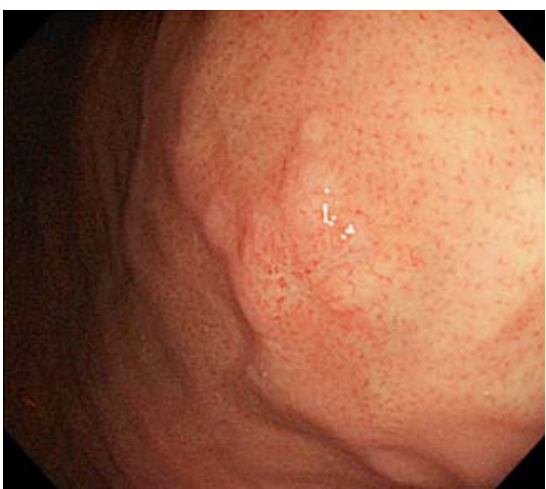

Fig. 1 Endoscopic view under white light showing a 15-mm diameter, flat, pale-colored, nodular lesion covered by mildly inflamed mucosa within the gastric body.

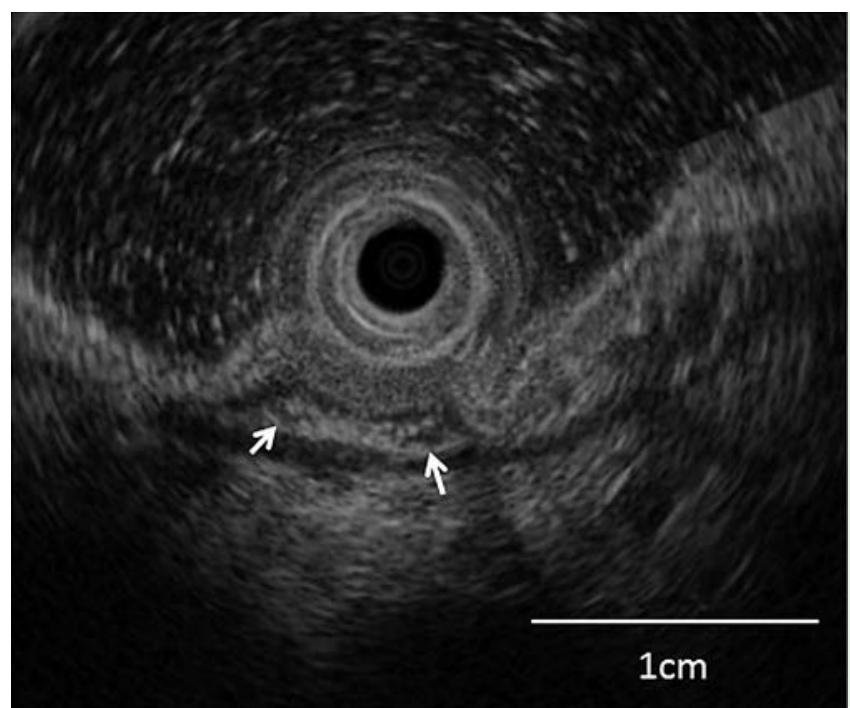

Fig. 2 Endoscopic ultrasound (EUS) image of the gastric lesion showing a thickened mucosal layer with small hypoechoic areas (arrows) in the submucosa.
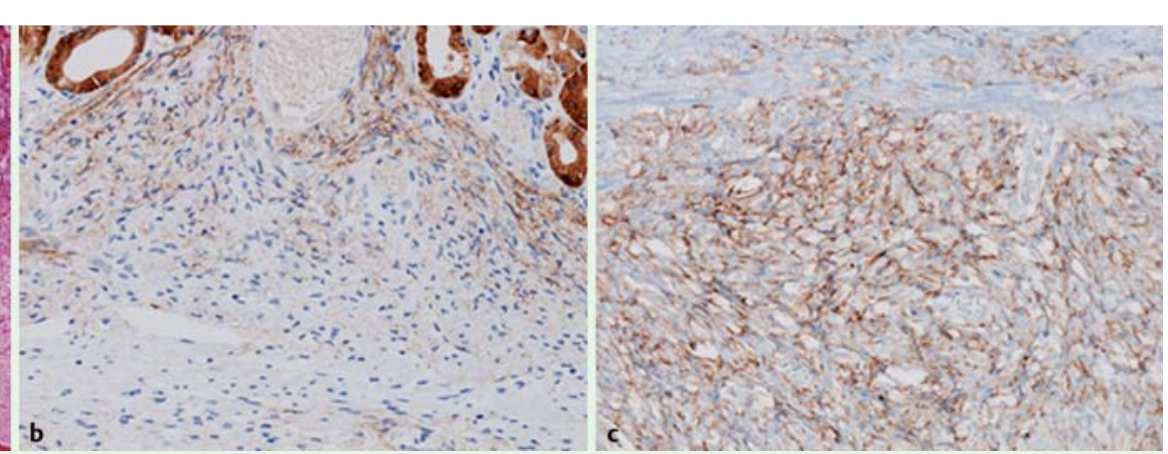

Fig. 3 Histopathological examination of the samples obtained by endoscopic submucosal dissection (ESD) of the lesion showing: a focal proliferation of spindle cells without atypia from the lamina propria to the submucosa (low power view); b weak positivity for EMA by immunohistochemical staining; c strong positivity for claudin-1 by immunohistochemical staining, consistent with the diagnosis of a perineurioma. 
Competing interests: None

N. Muguruma', S. Okamura', Y. Imoto', T. Sueuchi ${ }^{1}$, K. Okamoto ${ }^{1}$, H. Fujimoto ${ }^{2}$, K. Arita ${ }^{1}$, T. Hirose ${ }^{3}$, T. Takayama ${ }^{1}$

${ }^{1}$ Department of Gastroenterology and Oncology, The University of Tokushima Graduate School, Tokushima City, Japan

${ }^{2}$ Department of Internal Medicine, Health Insurance Naruto Hospital, Naruto, Japan

${ }^{3}$ Department of Diagnostic Pathology, Tokushima Prefectural Central Hospital, Tokushima City, Japan

\section{References}

1 Hornick JL, Fletcher CDM. Intestinal perineuriomas - Clinicopathologic definition of a new anatomic subset in a series of 10 cases. Am J Surg Pathol 2005; 29: 859-865

2 Lazarus SS, Trombetta LD. Ultrastructural identification of a benign perineurial cell tumor. Cancer 1978; 41: 1823-1829

3 Agaimy A, Wuensch PH. Perineurioma of the stomach: a rare spindle cell neoplasm that should be distinguished from gastrointestinal tumor. Pathol Res Pract 2005; 201: 463-467

4 Chetty $R$. Myxoid perineurioma presenting as a gastric polyp. Ann Diagn Pathol 2010; 14: $125-128$
Bibliography

DOI http://dx.doi.org/

10.1055/s-0031-1291748

Endoscopy 2012; 44: E182-E183

(c) Georg Thieme Verlag KG

Stuttgart · New York

ISSN 0013-726X

\section{Corresponding author}

\section{N. Muguruma, MD, PhD}

Department of Gastroenterology and Oncology The University of Tokushima Graduate School

3-18-15, Kuramoto-cho

Tokushima city 770-8503

Japan

Fax: +81-88-6339235

muguruma.clin.med@gmail.com 